\title{
Mouse model reveals root of breathing issues in rare form of autism
}

\section{BY ANNA GOSHUA}

5 NOVEMBER 2021

The breathing troubles seen in children with Pitt-Hopkins syndrome, a rare form of autism, stem from dysfunctional circuits and a rogue sodium channel in the brainstem, according to a new mouse study.

About 250 people worldwide have Pitt-Hopkins syndrome, which is characterized by intellectual disability, developmental delay, seizures and hyperactivity. Up to 50 percent of children with the syndrome also develop breathing problems in which they alternate between breathing rapidly and holding their breath.

"That type of breathing can easily contribute to aspiration pneumonia, which is a leading cause of death in Pitt-Hopkins," says lead investigator Daniel Mulkey, professor of physiology and neurobiology at the University of Connecticut in Storrs. It can also interfere with daily activities and cause children to fall or faint.

The syndrome arises from mutations in a gene called TCF4, which encodes a protein that helps turn other genes on or off. But scientists haven't been able to explain how that compromised gene translates into specific traits.

The new work, published in October in Nature Communications, traces the condition's hallmark breathing problems to a set of neurons in the brainstem and the presence of a sodium channel not usually found in the brain - although there are likely additional causes, the researchers say.

'One of the big questions that we've had all along is, 'How do we treat these problems?' because we don't know where they're coming from or what's causing them," says Kimberly Goodspeed, assistant professor of pediatrics at the University of Texas Southwestern in Dallas, who was not involved with the work. 


\section{Spectrum | Autism Research News}

https://www.spectrumnews.org

The rogue sodium channel, she and others say, could serve as a target for treatment.

Motion picture: Mice with a mutation in the TCF4 gene (right) move around their cages more than wildtype mice do (left).

\section{Mutated mice:}

Mulkey's team studied mice with a mutated copy of TCF4. Like people with Pitt-Hopkins syndrome, the animals are hyperactive and have disordered breathing, the researchers found. The mice also show fewer anxiety-like behaviors than expected in response to certain stimuli.

The team examined neurons in brain slices taken from the ventral parafacial region, an area of the brainstem that regulates breathing. Neurons that control inhalations could not communicate properly with the rest of the breathing circuitry, the researchers found. And 70 percent of a subset of neurons thought to be responsible for exhalations was missing.

The brain slices also contained NaV1.8, a sodium channel that is usually found only in peripheral nerves. Sodium channels help propagate electrical impulses, or action potentials, through neurons.

Treating the TCF4-mutant mice with a sodium-channel blocker that can enter the brain eased their breathing difficulties, hyperactivity and anxiety-like behavior. Knocking out the sodium channel in neurons that control breathing similarly improved the animals' breathing. The researchers did not examine behavioral traits in this experiment.

The researchers aren't certain exactly how the sodium channels contribute to symptoms of PittHopkins syndrome. It may be that "when those cells express this sodium channel, they fail to continue making action potentials and so they can't maintain their activity," Mulkey says.

The sodium channel offers a "specific molecular target that could potentially rescue some of these respiratory problems," says Eric Levine, professor of neuroscience at the University of Connecticut, who was not involved with the study.

\section{Fresh breath:}

The researchers plan to explore how the lack of TCF4 leads to the loss of certain cells in the breathing circuitry.

"I want to identify genes that are specific for these expiratory cells. Who are they, what are they doing, who do they talk to?" Mulkey says. 


\section{Spectrum | Autism Research News}

https://www.spectrumnews.org

But it's also important to look beyond this specific set of neurons at the broader context in which they operate, he says. "They don't function alone. They live in a neighborhood that includes astrocytes. It includes regulation of vascular tone. It includes inhibitory neurons that are all working in a sort of orchestra to control each other and influence the behavior."

Questions also remain about whether there are similarities between Pitt-Hopkins syndrome and other autism-related conditions, such as Rett syndrome, which involves a similar pattern of disordered breathing. Although it's unlikely that the cellular and molecular causes of these breathing problems are the same, they may share common downstream effects.

"Even if the underlying causes of those symptoms are different, they may converge on a similar downstream target, such as this sodium channel," Levine says. "That could turn out to be a common factor that could be targeted in these conditions and others as well."

Cite this article: https://doi.org/10.53053/LUYL3482 